\title{
Near-infrared fluorescence imaging of thoracic duct anatomy and function in open surgery and video-assisted thoracic surgery
}

\author{
Yoshitomo Ashitate, MD, ${ }^{\mathrm{a}, \mathrm{c}}$ Eiichi Tanaka, MD, PhD, ${ }^{\mathrm{c}}$ Alan Stockdale, MEd, ${ }^{\mathrm{a}}$ Hak Soo Choi, PhD, ${ }^{\mathrm{a}}$ and \\ John V. Frangioni, MD, $\mathrm{PhD}^{\mathrm{a}, \mathrm{b}}$
}

Objective: Chylothorax resulting from thoracic duct damage is often difficult to identify and repair. We hypothesized that near-infrared fluorescent light could provide sensitive, real-time, high-resolution intraoperative imaging of thoracic duct anatomy and function.

\begin{abstract}
Methods: In 16 rats, 4 potential near-infrared fluorescent lymphatic tracers were compared in terms of signal strength and imaging time: indocyanine green, the carboxylic acid of IRDye 800CW (LI-COR, Lincoln, $\mathrm{Neb}$ ), indocyanine green adsorbed to human serum albumin, and IRDye 800CW conjugated covalently to human serum albumin. Optimal agent was validated in 8 pigs approaching human size $(\mathrm{n}=6$ by open surgery with FLARE imaging system [Beth Israel Deaconess Medical Center, Boston, Mass] and $n=2$ by video-assisted thoracoscopic surgery minimally invasive [m-FLARE] imaging system [Beth Israel Deaconess Medical Center]). Lymphatic tracer injection site, dose, and timing were optimized.
\end{abstract}

Results: For signal strength, sustained imaging time, and clinical translatability, the best lymphatic tracer was indocyanine green, which is already Food and Drug Administration approved for other indications. In pigs, a simple subcutaneous injection of indocyanine green into lower leg $(\geq 36 \mu \mathrm{g} / \mathrm{kg})$, provided thoracic duct imaging with onset of about 5 minutes after injection, sustained imaging for at least 60 minutes after injection, and signal-to-background ratio of at least 2 . With this technology, normal thoracic duct flow, collateral flow, injury models, and repair models could all be observed under direct visualization.

Conclusions: Near-infrared fluorescent light could provide sensitive, sustained, real-time imaging of thoracic duct anatomy and function during both open and video-assisted thoracoscopic surgery in animal models. (J Thorac Cardiovasc Surg 2011;142:31-8)

\section{Supplemental material is available online.}

Thoracic duct injury, a rare but serious complication after chest surgery and major neck dissections, carries a high mortality in untreated patients. ${ }^{1-3}$ Intraoperative identification of the thoracic duct can be difficult, especially during

\footnotetext{
From the Division of Hematology/Oncology, ${ }^{\text {a }}$ Department of Medicine, and the Department of Radiology, ${ }^{\mathrm{b}}$ Beth Israel Deaconess Medical Center, Boston, Mass; and the Division of Cancer Diagnostics and Therapeutics, ${ }^{c}$ Hokkaido University Graduate School of Medicine, Sapporo, Japan.

Funded by National Institutes of Health (National Cancer Institute) Bioengineering Research Partnership grant \#R01-CA-115296 (to J.V.F.).

Disclosures: All FLARE technology is owned by Beth Israel Deaconess Medical Center, Boston, Mass, a teaching hospital of Harvard Medical School. As inventor, J.V.F. may someday receive royalties if products are commercialized. J.V.F. is the founder and unpaid director of The FLARE Foundation, a nonprofit organization focused on promoting the dissemination of medical imaging technology for research and clinical use.

Received for publication Nov 29, 2010; revisions received Feb 7, 2011; accepted for publication March 1, 2011; available ahead of print April 8, 2011.

Address for reprints: John V. Frangioni, MD, PhD, Beth Israel Deaconess Medical Center, Room SL-B05, 330 Brookline Ave, Boston, MA 02215 (E-mail: jfrangio@bidmc.harvard.edu).

$0022-5223 / \$ 36.00$

Copyright (C) 2011 by The American Association for Thoracic Surgery

doi:10.1016/j.jtcvs.2011.03.004
}

reoperation. Because traditional conservative treatment of thoracic duct injury has a high failure rate, ${ }^{1}$ intraoperative image guidance is essential for proper surgical management. ${ }^{4}$

Currently, lymphangiography and lymphoscintigraphy are available to preoperatively identify the site of thoracic duct damage; however, these techniques do not permit precise localization of the injured site. ${ }^{5-7}$ In addition, preoperative anatomic information is difficult to transfer directly to the intraoperative situation. In the past, blue dyes or indocyanine green (ICG) injections have been used, ${ }^{8-10}$ as well as an invasive injection procedure in which popliteal lymph node, testicular parenchyma, or the mesenteric lymph duct was used as the injection site. Although oral administration of heavy cream before surgery is sometimes performed to visualize chylous leakage or to prevent injury to the thoracic duct, the contrast provided is low and identification of the thoracic duct can be difficult. ${ }^{11}$ Optical imaging with near-infrared (NIR) fluorescence has the potential to solve all of these problems (as reviewed in Frangioni ${ }^{12}$ and by Gioux et $\mathrm{al}^{13}$ ). Indeed, thoracic duct NIR fluorescence imaging with ICG has been reported previously for the detection of chylous fistulas in the thoracic cavity. ${ }^{14}$

Intraoperative, real-time NIR fluorescence imaging has been used in many areas of surgery to visualize surgical 


\section{Abbreviations and Acronyms \\ CW800-CA = IRDye 800CW carboxylate \\ FDA $=$ Food and Drug Administration \\ FI $=$ fluorescence intensity \\ FLARE = Fluorescence-Assisted Resection and Exploration [system] \\ HSA $=$ human serum albumin \\ HSA800 = IRDye 800CW conjugated with human serum albumin \\ ICG = indocyanine green \\ ICG-HSA = indocyanine green adsorbed to human serum albumin \\ $\mathrm{m}$-FLARE $=$ Minimally Invasive Fluorescence- \\ Assisted Resection and \\ Exploration [system] \\ NIR = near-infrared \\ SBR}

anatomy and tissue function simultaneously and noninvasively with high spatial resolution. The enabling technology for our study is the Fluorescence-Assisted Resection and Exploration (FLARE) NIR optical imaging system (Beth Israel Deaconess Medical Center, Boston, Mass) ${ }^{15}$ which provides simultaneous acquisition of color video and 2 independent channels of invisible NIR fluorescence, 1 centered at $700 \mathrm{~nm}$ and the other at $800 \mathrm{~nm}$, and the new, minimally invasive version of FLARE, (m-FLARE), for video-assisted thoracoscopic surgery. In this study, we hypothesized that these NIR imaging systems might provide adequate contrast for anatomic and functional assessment of the thoracic duct after a simple subcutaneous injection. We also sought to optimize the lymphatic tracer, injection site, dose, and timing for the intraoperative assessment of the thoracic duct.

\section{MATERIALS AND METHODS Lymph Tracer Preparation}

Pharmaceutical grade ICG was purchased from Akorn (Akorn, Incorporated, Lake Forest, Ill). ICG has been widely used in such clinical applications as liver function testing, cardiac output monitoring, and ophthalmic angiography. Recently, it has also been used in image-guided surgery studies to assess vessel patency after anastomoses in coronary artery bypass grafting ${ }^{16}$ and to evaluate perforator location and flap perfusion in breast reconstructive surgery after mastectomy. ${ }^{17}$ The carboxylic acid form of IRDye 800CW (CW800-CA) was purchased from LI-COR Biotechnology (Lincoln, Neb). Both were diluted in phosphate-buffered saline solution ( $\mathrm{pH}$ 7.4) before injection. The noncovalent adsorption of ICG to human serum albumin (HSA), at a molar ratio of 1:1 (ICG-HSA), was performed as described previously. ${ }^{18}$ The covalent conjugation of IRDye $800 \mathrm{CW}$ to HSA at a fluorophore to protein substitution ratio of 3.0 (HSA800) was prepared as described previously. ${ }^{18}$ The optical properties of all agents were measured in fetal bovine serum. The quantum yields of contrast agents in fetal bovine serum were calculated with ICG in dimethyl sulfoxide (quantum yield $13 \%{ }^{19}$ ) as the calibration standard. For in vitro optical property measurements, online fiberoptic HR2000 absorbance (200-1100 nm) and USB2000FL fluorescence $(350-1000 \mathrm{~nm}$ ) spectrometers (Ocean Optics, Inc, Dunedin, Fla) were used. NIR excitation was provided by a 770-nm NIR laser diode light source (Electro Optical Components, Inc, Santa Rosa, Calif) set to $8 \mathrm{~mW}$ and coupled through a $300-\mu \mathrm{m}$ core diameter, 0.22 NA fiber (Fiberguide Industries Inc, Stirling, NJ).

\section{Animal Studies}

Animal studies were performed under the supervision of approved institutional protocols. Sixteen male $300-\mathrm{g}$ Sprague-Dawley rats (Taconic Farms, Germantown, NY) were anesthetized with $65-\mathrm{mg} / \mathrm{kg}$ intraperitoneal pentobarbital and were ventilated by tracheotomy. Eight female Yorkshire pigs (E.M. Parsons and Sons, Hadley, Mass) averaging $36.1 \mathrm{~kg}$ were induced with 4.4-mg/kg intramuscular tiletamine hydrochloride and zolazepam hydrochloride (Telazol; Fort Dodge Animal Health, Fort Dodge, Iowa), intubated, and maintained with $2 \%$ isoflurane. Selective ventilation of the left lung was used to deflate the right lung and help provide better visualization of the thoracic duct. Electrocardiogram, heart rate, oxygen saturation, and body temperature were monitored throughout the experiment.

\section{Optimization of Lymphatic Tracer in Rat}

In 16 anesthetized rats, $50 \mu \mathrm{L}$ of $10-\mu \mathrm{mol} / \mathrm{L}$ contrast agent $(1.5 \mu \mathrm{g} / \mathrm{kg}$ administered dose) was injected into mesenteric lymph nodes. For visualization of the thoracic duct, a right thoracotomy was performed under controlled ventilation to expose the posterior mediastinum near the spinal column. The FLARE imaging system ${ }^{15}$ was used for quantification of NIR fluorescence images. Intensities of the thoracic duct were measured at 3, 5, 10, and 15 minutes after injection. The signal-to-background ratio (SBR) was defined as the NIR fluorescence intensity (FI) of a region of interest over the thoracic duct divided by that of an equivalent size and shape region of interest over the thoracic wall.

\section{Optimization of ICG Injection Site, Dose, and Imaging Time in Pig}

Eight pigs were anesthetized as described previously and placed in a left lateral decubitus position. A right thoracotomy was performed for open surgery $(\mathrm{n}=6)$. Three small $(2-3 \mathrm{~cm})$ incisions were made in the right costal interspaces for video-assisted thoracoscopic surgery $(n=2)$. The incision positions included the midaxillary line at the 5th intercostal space, anterior axillary line at the 7th intercostal space, and posterior axillary line at the 7th intercostal space. The thoracoscope and surgical instruments were inserted through these incisions. An additional incision was made to retract the lung lobe or diaphragm if necessary.

After the inferior pulmonary ligament was divided, the thoracic duct was visualized with the FLARE or m-FLARE imaging systems. Fluorophore was injected subcutaneously into the groin (near, but not in groin lymph nodes), the thigh (near the great saphenous vein detected by ultrasonography or palpation), and the shin (near the great saphenous vein). Control injections were made directly into groin lymph nodes exposed by dissection. Fluorophore injections ( $\mathrm{n}=3$ per condition) could be repeated every 2 hours to minimize the number of animals needed. In 3 pigs per condition, $50 \mathrm{~g}$ of heavy cream was administered into the stomach or $1 \mathrm{cc}$ of undiluted methylene blue was administered directly into a groin lymph node, and the thoracic duct was imaged over time.

To assess the affect of injected dose on SBR, the specified doses were injected into the optimal injection site $(n=3$ per dose). After each injection, gentle massage was performed for 5 minutes to cause smooth migration of fluorophore. For quantitative assessment, images were recorded at the time of injection ( $T=0$ minutes), then at $5,10,15,30,45$, and 60 minutes after injection. At each time point, the FI of a region of interest over the thoracic duct and thoracic wall were quantified with custom software. The 
TABLE 1. Physical and optical properties of near-infrared fluorescent lymphatic tracers in fetal bovine serum

\begin{tabular}{lcccccc}
\hline $\begin{array}{c}\text { Lymphatic } \\
\text { tracer }\end{array}$ & $\begin{array}{c}\text { Molecular } \\
\text { weight }\end{array}$ & $\begin{array}{c}\text { Hydrodynamic } \\
\text { diameter }\end{array}$ & $\begin{array}{c}\text { Peak } \\
\text { absorbance }\end{array}$ & $\begin{array}{c}\text { Peak } \\
\text { fluorescence }\end{array}$ & $\begin{array}{c}\text { Quantum } \\
\text { yield* }\end{array}$ & Description \\
\hline ICG & $776 \mathrm{Da}$ & $1.2 \mathrm{~nm}$ & $807 \mathrm{~nm}$ & $822 \mathrm{~nm}$ & $9.3 \%$ & $\begin{array}{c}\text { Small molecule, FDA approved for cardiac and hepatic } \\
\text { function studies }\end{array}$ \\
CW800-CA & $1069 \mathrm{Da}$ & $1.3 \mathrm{~nm}$ & $786 \mathrm{~nm}$ & $800 \mathrm{~nm}$ & $14.2 \%$ & $\begin{array}{c}\text { Tetra-sulphonated heptamethine indocyanine small } \\
\text { molecule with improved quantum yield }\end{array}$ \\
ICG-HSA & $67 \mathrm{kDa}$ & $7.3 \mathrm{~nm}$ & $800 \mathrm{~nm}$ & $814 \mathrm{~nm}$ & $9.4 \%$ & $\begin{array}{c}\text { Noncovalent adsorption of ICG to human serum albumin } \\
\text { (1:1 molar ratio) }\end{array}$ \\
HSA800 & $70 \mathrm{kDa}$ & $7.4 \mathrm{~nm}$ & $784 \mathrm{~nm}$ & $802 \mathrm{~nm}$ & $12.1 \%$ & $\begin{array}{c}\text { Covalent conjugation of CW800 to human serum } \\
\text { albumin }(3: 1 \text { molar ratio) }\end{array}$ \\
\hline
\end{tabular}

ICG, Indocyanine green; FDA, Food and Drug Administration; $C W 800-C A$, IRDye 800CW carboxylate; ICG- $H S A$, indocyanine green adsorbed to human serum albumin; HSA 800 , IRDye $800 \mathrm{CW}$ conjugated with human serum albumin. *Quantum yield in fetal bovine serum supplemented with 50 mM 4-(2-hydroxyethyl)-1-piperazineethanesulfonic acid ( $\mathrm{pH} 7.4)$, relative to ICG in dimethyl sulfoxide (13\%).

performance metric was the SBR, where the thoracic wall was used as background, derived as SBR $=$ ROIFI/ BGFI, where ROIFI indicates the FI of the region of interest and BGFI denotes the FI of the background. For the injury and treatment model, the pleura between the azygos vein and the spine was opened and the thoracic duct was exposed. Next, thoracic duct injury was induced with a 20 -gauge spinal needle and repaired by ligation with a 3-0 silk suture above and below the injury site.

\section{NIR Fluorescence Imaging System}

The FLARE imaging system has been described in detail previously. ${ }^{20}$ Our experiments used 40,000 lux of white light (400-650 nm), $4.0 \mathrm{~mW}$ / $\mathrm{cm}^{2}$ of $670-\mathrm{nm}$ NIR fluorescence excitation light, and $11.0 \mathrm{~mW} / \mathrm{cm}^{2}$ of 760-nm NIR fluorescence excitation light. NIR fluorescence camera exposure time was $67 \mathrm{~ms}$ or shorter. In the color-NIR merge images, $800-\mathrm{nm}$ NIR fluorescence was pseudocolored green and overlaid on top of the color video image.

\section{m-FLARE Imaging System Components}

The initial endoscopic imaging system has been described in detail previously. ${ }^{21,22}$ The current version of m-FLARE introduced in this study used white light from a custom 300-W xenon light source (Wilson Associates, Weymouth, Mass) equipped with filtration to remove all NIR, NIR excitation light from a $1-\mathrm{W} 670-\mathrm{nm}$ and $2.5-\mathrm{W}$ 760-nm laser diode assembly (OZ Optics, Ottawa, Ontario, Canada) combined with a custom light mixer (Qioptiq Imaging Solutions, Fairport, NY) and a 0.6-NA fiber optic cable connected to a standard rigid laparoscope $\left(10 \mathrm{~mm}\right.$ diameter, $0^{\circ}$; Karl Storz $\mathrm{GmbH} \&$ Co. KG, Tuttlingen, Germany). Optimal distance between laparoscope and the surgical field was $10 \mathrm{~cm}$. Through the laparoscope, 30,000 lux of white light $(400-650 \mathrm{~nm}), 6.0 \mathrm{~mW} / \mathrm{cm}^{2}$ of $670-\mathrm{nm}$ NIR fluorescence excitation light, and $20.0-\mathrm{mW} / \mathrm{cm}^{2}$ of $760-\mathrm{nm}$ NIR fluorescence excitation light were generated over a 5-cm diameter field of view. The eyepiece of the laparoscope was attached to custom optics (Qioptiq
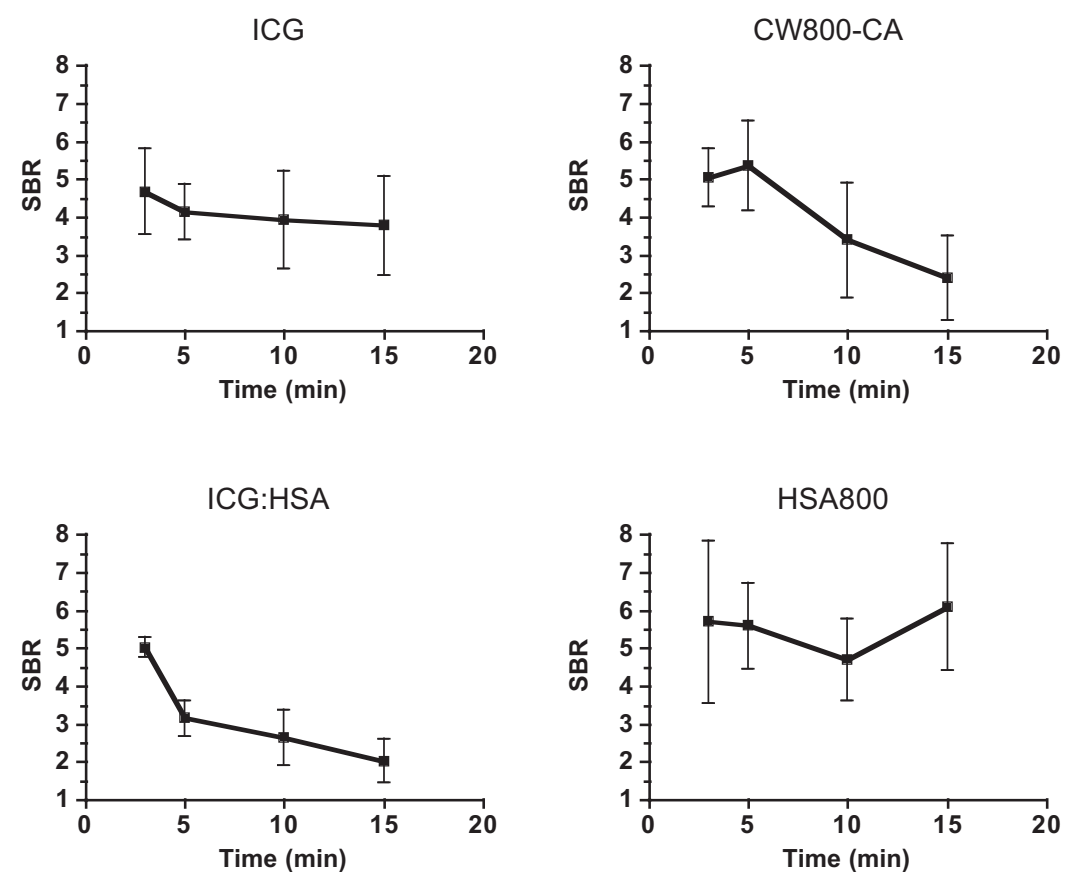

FIGURE 1. Quantitative assessment of various near-infrared lymphatic tracers for thoracic duct imaging. Signal-to-background ratio ( $S B R$, mean \pm SEM) over time in the thoracic duct of rats after injection of $1.5-\mu \mathrm{g} / \mathrm{kg}$ doses of the specified lymphatic tracers into mesenteric lymph nodes $(\mathrm{n}=4 \mathrm{independent}$ animals for each contrast agent studied). ICG, Indocyanine green; $C W 800-C A$, IRDye $800 \mathrm{CW}$ carboxylate; ICG-HSA, indocyanine green adsorbed to human serum albumin; HSA800, IRDye $800 \mathrm{CW}$ conjugated with human serum albumin. 

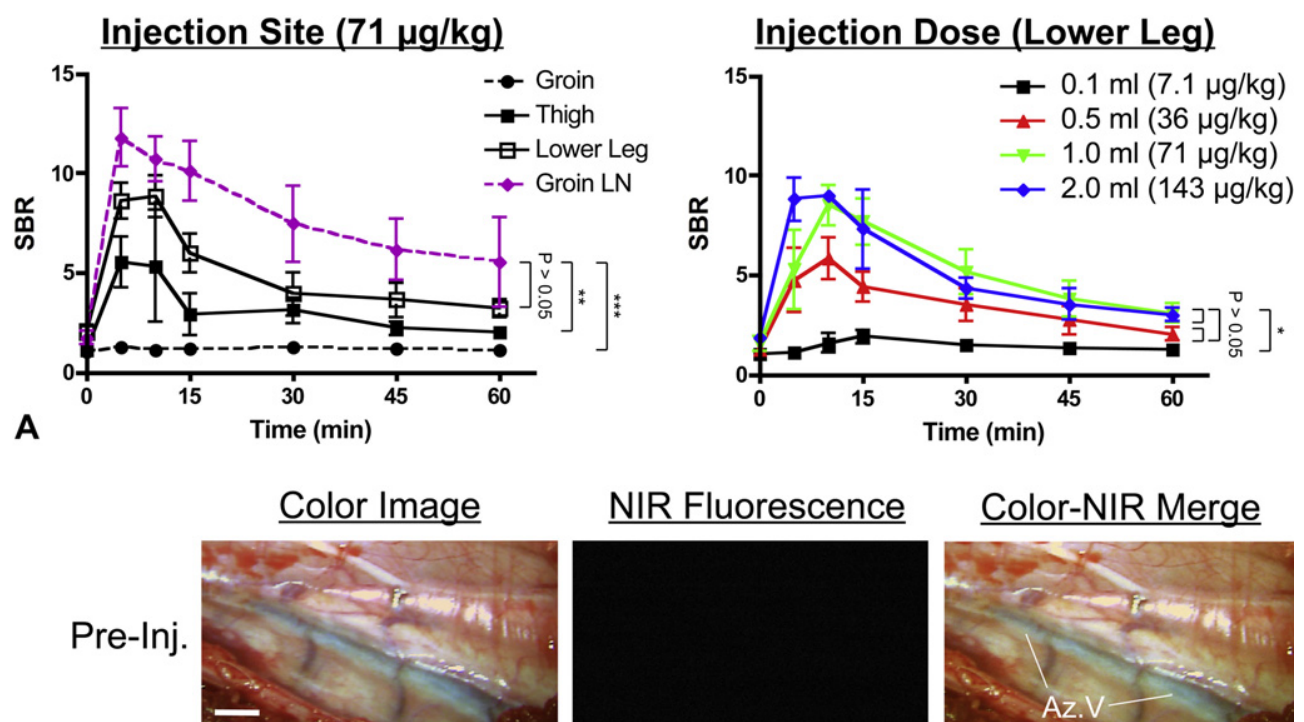

\section{Color-NIR Merge}
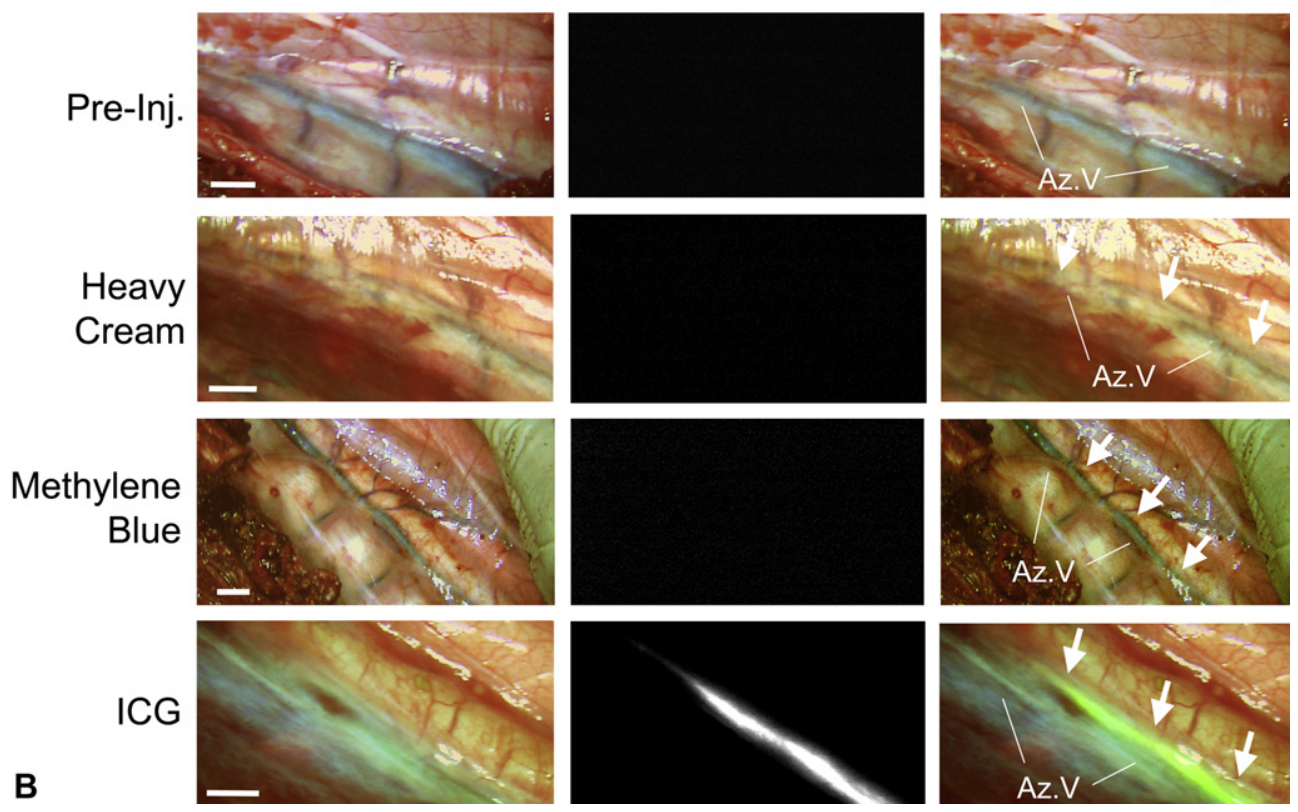

FIGURE 2. Thoracic duct imaging during open surgery in pigs. A, Signal-to-background ratio ( $S B R$, mean \pm SEM) of the thoracic duct over time for different injection sites (left, $\mathrm{n}=3$ per condition) and different injection doses (right, $\mathrm{n}=3$ per condition). Statistical assessments of the groin lymph node $(L N)$ versus the lower leg, the groin lymph node versus the thigh, and the groin lymph node versus the groin subcutaneum (left) are shown. Statistical assessment of the various doses $(36,71$, and $143 \mu \mathrm{g} / \mathrm{kg}$ ) and the difference between $7.1 \mu \mathrm{g} / \mathrm{kg}$ and $71 \mu \mathrm{g} / \mathrm{kg}$ (right) are also shown. Asterisk indicates $P<.05$; double asterisk indicates $P<.01$; and triple asterisk indicates $P<.001$, all by Tukey multiple comparison test. B, Comparison of typical contrast achieved with orally administered heavy cream, methylene blue, and indocyanine green (ICG). Before injection (Pre-inj., top row), the thoracic duct is not visible. After heavy cream (second row), the thoracic duct (white arrows) appears milky white. After methylene blue injection into a groin lymph node (third row), the thoracic duct (white arrows) has a bluish tint, much like the azygos vein $(A z . V)$. After subcutaneous injection of $71 \mu \mathrm{g} / \mathrm{kg}$ of indocyanine green into the lower leg (bottom row), the thoracic duct (arrows) is visualized in high contrast with near-infrared (NIR) fluorescence. Shown are representative images from $\mathrm{n}=3$ pigs per condition: color video (left), near-infrared fluorescence (middle), and a pseudocolored (lime green) merge (right). Scale bar represents $1 \mathrm{~cm}$.

Imaging Solutions), which permitted simultaneous acquisition of color video and NIR light with a scA640-74gc (Basler AG, Ahrensburg, Germany) color charge-coupled device camera and a GC655 (Prosilica, Stadtroda, Germany) NIR camera. Only the 800-nm emission channel was needed to image ICG.

\section{Statistical Analysis}

An analysis of variance and Tukey multiple comparison test were used to determine statistical differences between multiple groups. Tukey multiple comparison test and analysis of variance were used to show the pair that displayed significant difference among the group. The analyzed values are described as mean \pm SEM. All analyses were performed with GraphPad Prism software (version 4.0; GraphPad Software Inc, La Jolla, Calif).

\section{RESULTS \\ Optical Properties}

The physical and optical properties of the NIR fluorescent lymphatic tracers tested for thoracic duct imaging are detailed in Table 1. ICG is a disulfonated heptamethine indocyanine small molecule that is already approved by the Food and Drug Administration (FDA) for cardiac output and hepatic function studies, as well as for ophthalmic angiography. It has an extremely small hydrodynamic diameter, which allows it to travel through lymph nodes and into the thoracic duct. CW800-CA is a new and improved 


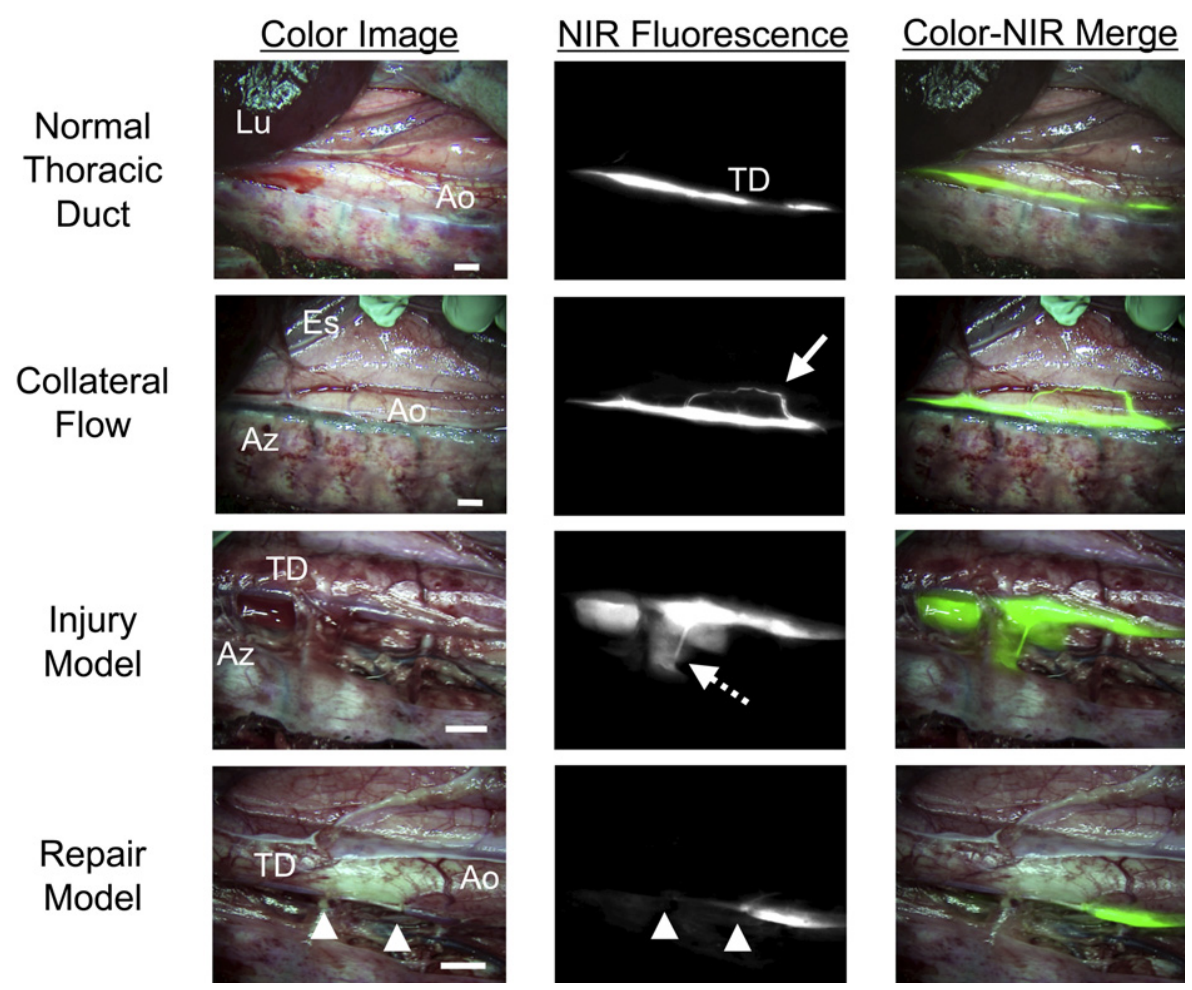

FIGURE 3. High-resolution imaging of thoracic duct anatomy and function. Shown are representative images 30 minutes after injection of $36-\mu \mathrm{g} / \mathrm{kg}$ indocyanine green into the lower leg of pigs. Conditions included normal flow (top row), collateral flow (second row, solid arrow), an injury model (third row, dotted arrow), and image-guided repair of the thoracic duct (bottom row, arrowheads). Shown are color video (left), near-infrared (NIR) fluorescence (middle), and a pseudocolored (lime green) merge (right). Lu, Lung; Ao, aorta; TD, thoracic duct; Es, esophagus; Az, azygos vein. Scale bar represents $1 \mathrm{~cm}$.

heptamethine indocyanine small molecule with higher quantum yield and lower protein binding. ${ }^{18}$ ICG-HSA is the adsorption of ICG to a moderate-affinity binding site on HSA. This complex is stable enough to permit sentinel lymph node mapping, but at only $7.3 \mathrm{~nm}$ in hydrodynamic diameter it can also pass through lymph nodes over time. ${ }^{18}$ We studied this complex with the hope that a larger hydrodynamic diameter would slow the rate of travel through the lymphatic system and permit thoracic duct imaging for a longer period. HSA800 is an improved NIR fluorescent albumin derivative with higher quantum yield and stability. ${ }^{18}$

\section{Thoracic Duct Imaging and Lymphatic Tracer Performance in Rats}

We found several differences among the NIR fluorescent contrast agents with respect to SBR of the thoracic duct and the period of optimal visualization. With a 15-minute visualization period, ICG demonstrated SBR values of 4 to 5 , which remained relatively constant (Figure 1). Despite a higher quantum yield of CW800-CA, which is supposed to cause higher FI, or the larger hydrodynamic diameter of ICG-HSA, the SBR values of both at 3 minutes after injection were close to that of ICG and decayed significantly during the 15-minute imaging interval (Figure 1). These results are likely explained by rapid uptake into the bloodstream (CW800-CA) or desorption of ICG from HSA (ICG-HSA). We found that HSA800 showed the highest SBR values, although not statistically significantly different from those of ICG. Because HSA800 is not available for clinical translation, ICG was used in all subsequent experiments.

\section{Quantitative Assessment of ICG Injection Site, Dose, and Imaging Period in Pigs}

Because mesenteric lymph node injection is not a reasonable clinical option, we attempted to find a more suitable injection site. We injected $1 \mathrm{~mL}$ of a 3.2-mmol/L ICG solution $(71 \mu \mathrm{g} /$ $\mathrm{kg}$ ) into the groin, thigh, or lower leg of pigs. As shown in Figure 2, $A$, injection into the groin worked poorly without advance exposure of groin lymph nodes and direct injection. Direct injection into already exposed groin lymph nodes served as a control and provided rapid and bright thoracic duct imaging (Figure 2, A). Simple subcutaneous injection into the thigh or lower leg (Figure 2, $A$, and Figure E1), however, provided acceptably high SBR values for as long as 120 minutes after injection (Figure E2). Although there was no statistically significant difference between the thigh and lower leg sites $(P>.05)$, the lower leg site produced a higher SBR and 


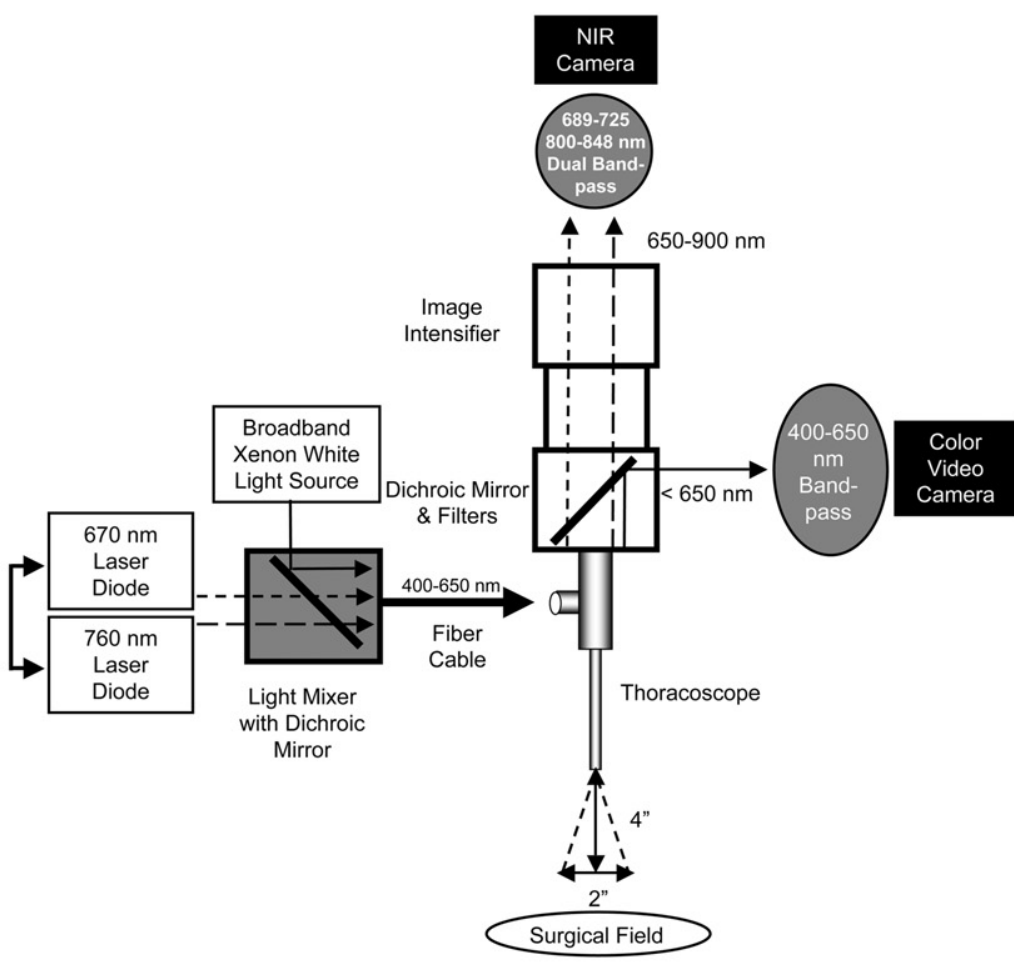

A
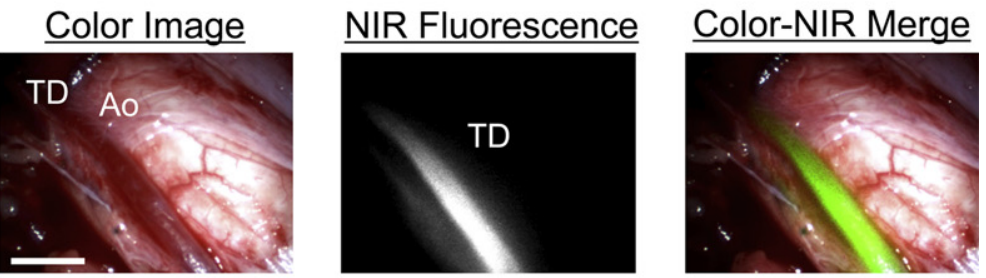

Collateral
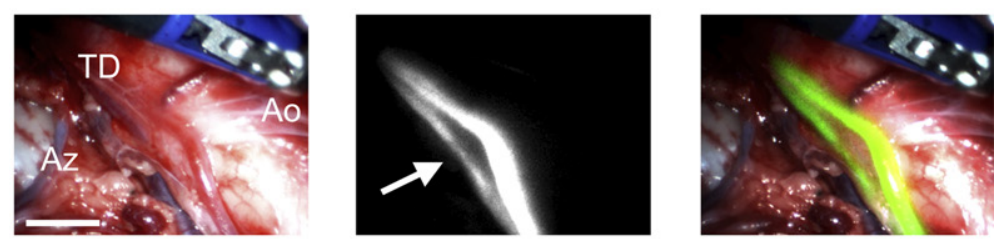

Flow
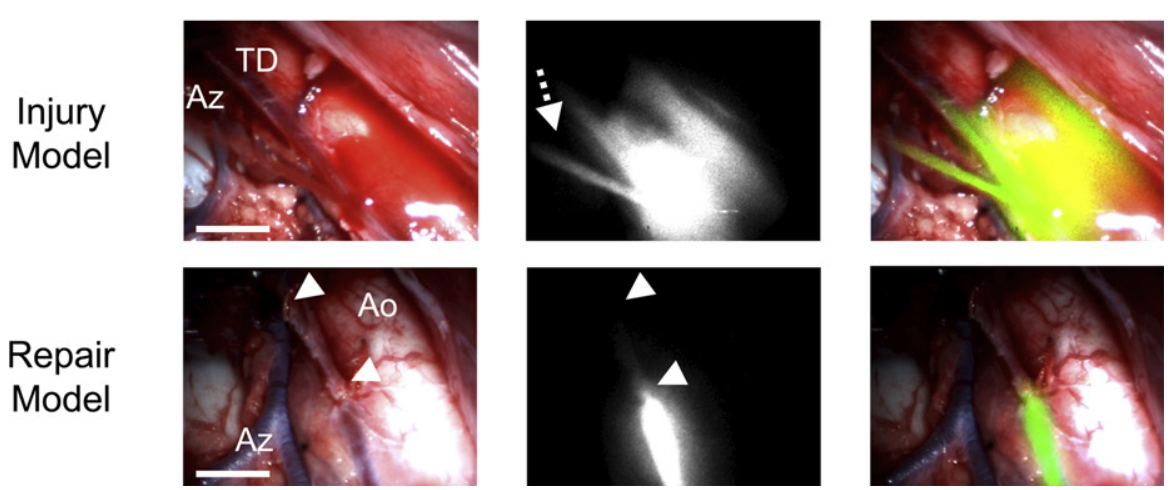

FIGURE 4. Near-infrared (NIR) fluorescence imaging of the thoracic duct with m-FLARE and video-assisted thoracoscopic surgery. A, Light paths and system components of the m-FLARE imaging system for video-assisted thoracoscopic surgery. B, Video-assisted thoracoscopic surgery-based m-FLARE imaging of the thoracic duct. Shown are representative images 30 minutes after injection of $36-\mu \mathrm{g} / \mathrm{kg}$ indocyanine green into the lower leg of pigs $(\mathrm{n}=2$ ). Conditions included normal flow (top row), collateral flow (second row, solid arrow), an injury model (third row, dotted arrow), and image-guided repair of the thoracic duct (bottom row, arrowheads). Shown are color video (left), near-infrared fluorescence (middle), and a pseudocolored (lime green) merge (right). TD, Thoracic duct, $A o$, aorta, $A z$, azygos vein. Scale bar represents $1 \mathrm{~cm}$. 
was chosen for further study. In comparison with ICG, oral administration of heavy cream or injection of methylene blue directly into a groin lymph node provided relatively poor contrast (Figure 2, B). In the latter case, differentiation of the thoracic duct from the azygos vein was difficult.

To optimize the dose, $0.1 \mathrm{~mL}$ of $3.2-\mathrm{mmol} / \mathrm{L}$ ICG $(7.1$ $\mu \mathrm{g} / \mathrm{kg}), 0.5 \mathrm{~mL}$ of $3.2-\mathrm{mmol} / \mathrm{L} \mathrm{ICG}(36 \mu \mathrm{g} / \mathrm{kg}), 1.0 \mathrm{~mL}$ of $3.2-\mathrm{mmol} / \mathrm{L} \mathrm{ICG}(71 \mu \mathrm{g} / \mathrm{kg})$, and $2.0 \mathrm{~mL}$ of $3.2-\mathrm{mmol} / \mathrm{L}$ ICG $(143 \mu \mathrm{g} / \mathrm{kg})$ were injected subcutaneously into the lower leg, and the thoracic duct was imaged over time. All doses provided SBR values of at least 2 except for the $7.1-\mu \mathrm{g} / \mathrm{kg}$ dose. Although SBR trended higher with increasing dose (Figure 2, $A$ ), there was no statistically significant difference among $36-\mu \mathrm{g} / \mathrm{kg}, 71-\mu \mathrm{g} / \mathrm{kg}$, and $143-\mu \mathrm{g} / \mathrm{kg}$ doses $(P>.05)$.

\section{Real-Time Imaging of Normal Flow, Injury, and Repair in the Thoracic Duct of Pigs}

With injection of $36-\mu \mathrm{g} / \mathrm{kg}$ of ICG into the lower leg, pulsatile flow and collateral flow within the thoracic duct could be assessed in real time (Figure 3). Similarly, if the thoracic duct was injured, intraoperative NIR fluorescence imaging provided immediate localization of the injury, and repair could be performed under image guidance (Figure 3). Additionally, in the color image, ICG did not stain the surgical field even after injury, because it was significantly diluted during the long migration from the injection site.

\section{NIR Fluorescence Video-Assisted Thoracoscopic Surgery Imaging of the Thoracic Duct}

The minimally invasive m-FLARE imaging system (Figure $4, A$ ) provided similar imaging capabilities to those seen during open surgery with FLARE. As shown in Figure $4, B$, m-FLARE permitted real-time visualization of the thoracic duct under conditions of normal flow, collateral flow, injury, and repair.

\section{DISCUSSION}

This study exploited invisible NIR fluorescent light and the contrast agent ICG, which is already FDA-approved for other indications, to provide surgeons with real-time, intraoperative identification of the thoracic duct during both open and minimally invasive surgical procedures. Currently, preoperative administration of heavy cream is used to detect fistulas during video-assisted thoracoscopic surgical ligation of the thoracic duct; however, heavy cream provides poor contrast (Figure 2, B). In contrast, a properly chosen NIR fluorescent lymphatic tracer, used in conjunction with an appropriate imaging system, can provide highly sensitive visualization.

In this study, ICG provided an excellent SBR and a long visualization time after a single subcutaneous injection of at least $36 \mu \mathrm{g} / \mathrm{kg}$ into the lower leg without changing surgical field. Because of the availability of NIR imaging systems, such as the FLARE, m-FLARE, SPY (Novadaq Technologies Inc, Mississauga, Ontario, Canada), ${ }^{16}$ and PDE (Hamamatsu Photonics, Hamamatsu, Japan), ${ }^{23}$ and the existing FDA approval for ICG in other indications, future clinical trials should focus on optimizing dose and injection site in patients with our data from swine as a starting point.

A key variable in lymphatic tracer optimization is the trade-off between contrast agent concentration at the injection site and dilution as the tracer flows through lymphatic channels. The higher the injected concentration, the lower the total fluorescence as a result of quenching. As the tracer travels through lymphatic channels and gets diluted in lymph, however, the NIR fluorescence signal becomes brighter and brighter. The key to successful imaging in patients will require delineation of a volume of ICG that does not cause tattooing at the injection site, a concentration of ICG that results in maximal SBR in the thoracic duct, and proper timing after injection for optimal thoracic duct contrast.

The best injection site in patients remains to be determined. The webbed space between the first and second toes of the foot is often used as the injection site for lymphangiography. ${ }^{24}$ Such a long distance from the thoracic duct will cause significant dilution of ICG, however, and thus will result in lower SBR. Injection into the upper leg could be problematic, however, because Schacht and colleagues ${ }^{25}$ have reported that there exists a narrow and deep plane of subcutaneous fat that is not present in the lower leg or dorsum of the foot. Such subcutaneous fat could impede the flow of ICG into lymphatic channels (see, for example, the groin injection in Figure 2, $A$ ).

In summary, we have demonstrated high-sensitivity, realtime visualization of the thoracic duct with invisible NIR fluorescence light. Imaging is performed with an agent already FDA approved for other indications, making translation to the clinic both feasible and practical.

We thank Rita G. Laurence for assistance with animal surgery, Lindsey Gendall and Lorissa A. Moffitt for editing, and Eugenia Trabucchi and Linda Keys for administrative assistance.

\section{References}

1. Lapp GC, Brown DH, Gullane PJ, McKneally M. Thoracoscopic management of chylous fistulae. Am J Otolaryngol. 1998;19:257-62.

2. Wurnig PN, Hollaus PH, Ohtsuka T, Flege JB, Wolf RK. Thoracoscopic direct clipping of the thoracic duct for chylopericardium and chylothorax. Ann Thorac Surg. 2000;70:1662-5.

3. Fahimi H, Casselman FP, Mariani MA, van Boven WJ, Knaepen PJ, van Swieten HA. Current management of postoperative chylothorax. Ann Thorac Surg. 2001;71:448-51.

4. Noel AA, Gloviczki P, Bender CE, Whitley D, Stanson AW, Deschamps C. Treatment of symptomatic primary chylous disorders. J Vasc Surg. 2001;34:785-91.

5. Sachs PB, Zelch MG, Rice TW, Geisinger MA, Risius B, Lammert GK. Diagnosis and localization of laceration of the thoracic duct: usefulness of lymphangiography and CT. AJR Am J Roentgenol. 1991;157:703-5.

6. Stavngaard T, Mortensen J, Brenoe J, Svendsen LB. Lymphoscintigraphy using technetium-99m human serum albumin in chylothorax. Thorac Cardiovasc Surg. 2002;50:250-2. 
7. Qureshy A, Kubota K, Ono S, Sato T, Fukuda H. Thoracic duct scintigraphy by orally administered I-123 BMIPP: normal findings and a case report. Clin Nucl Med. 2001;26:847-55.

8. Enwiller TM, Radlinsky MG, Mason DE, Roush JK. Popliteal and mesenteric lymph node injection with methylene blue for coloration of the thoracic duct in dogs. Vet Surg. 2003;32:359-64.

9. Tsuruno A, Shibazaki A, Kanda N, Shibata H. Visualization of the thoracic duct with injections of dyes or contrast media into the testicular parenchyma in the rabbit. J Vet Med Sci. 2009;71:759-62.

10. Nakayama H, Ito H, Kato Y, Tsuboi M. Ultrasonic scalpel for sealing of the thoracic duct: evaluation of effectiveness in an animal model. Interact Cardiovasc Thorac Surg. 2009;9:399-401.

11. Nair SK, Petko M, Hayward MP. Aetiology and management of chylothorax in adults. Eur J Cardiothorac Surg. 2007;32:362-9.

12. Frangioni JV. In vivo near-infrared fluorescence imaging. Curr Opin Chem Biol. 2003;7:626-34.

13. Gioux S, Choi HS, Frangioni JV. Image-guided surgery using invisible nearinfrared light: fundamentals of clinical translation. Mol Imaging. 2010;9:237-55.

14. Kamiya K, Unno N, Konno H. Intraoperative indocyanine green fluorescence lymphography, a novel imaging technique to detect a chyle fistula after an esophagectomy: report of a case. Surg Today. 2009;39:421-4.

15. Troyan SL, Kianzad V, Gibbs-Strauss SL, Gioux S, Matsui A, Oketokoun R, et al. The FLARE intraoperative near-infrared fluorescence imaging system: a first-inhuman clinical trial in breast cancer sentinel lymph node mapping. Ann Surg Oncol. 2009; 16:2943-52.

16. Reuthebuch O, Häussler A, Genoni M, Tavakoli R, Odavic D, Kadner A, et al. Novadaq SPY: intraoperative quality assessment in off-pump coronary artery bypass grafting. Chest. 2004;125:418-24.
17. Lee BT, Hutteman M, Gioux S, Stockdale A, Lin SJ, Ngo LH, et al. The FLARE intraoperative near-infrared fluorescence imaging system: a first-in-human clinical trial in perforator flap breast reconstruction. Plast Reconstr Surg. 2010;126: 1472-81.

18. Ohnishi S, Lomnes SJ, Laurence RG, Gogbashian A, Mariani G, Frangioni JV. Organic alternatives to quantum dots for intraoperative near-infrared fluorescent sentinel lymph node mapping. Mol Imaging. 2005;4:172-81.

19. Benson C, Kues HA. Absorption and fluorescence properties of cyanine dyes. J. Chem Eng Data. 1977;22:379-83.

20. Gioux S, Kianzad V, Ciocan R, Gupta S, Oketokoun R, Frangioni JV. High-power, computer-controlled, light-emitting diode-based light sources for fluorescence imaging and image-guided surgery. Mol Imaging. 2009;8: 156-65.

21. Matsui A, Tanaka E, Choi HS, Kianzad V, Gioux S, Lomnes SJ, et al. Real-time, near-infrared, fluorescence-guided identification of the ureters using methylene blue. Surgery. 2010;148:78-86.

22. Matsui A, Tanaka E, Choi HS, Winer JH, Kianzad V, Gioux S, et al. Realtime intra-operative near-infrared fluorescence identification of the extrahepatic bile ducts using clinically available contrast agents. Surgery. 2010; 148:87-95.

23. Unno N, Suzuki M, Yamamoto N, Inuzuka K, Sagara D, Nishiyama M, et al. Indocyanine green fluorescence angiography for intraoperative assessment of blood flow: a feasibility study. Eur J Vasc Endovasc Surg. 2008;35:205-7.

24. Guermazi A, Brice P, Hennequin C, Sarfati E. Lymphography: an old technique retains its usefulness. Radiographics. 2003;23:1541-60.

25. Schacht V, Luedemann W, Abels C, Berens von Rautenfeld D. Anatomy of the subcutaneous lymph vascular network of the human leg in relation to the great saphenous vein. Anat Rec (Hoboken). 2009;292:87-93. 

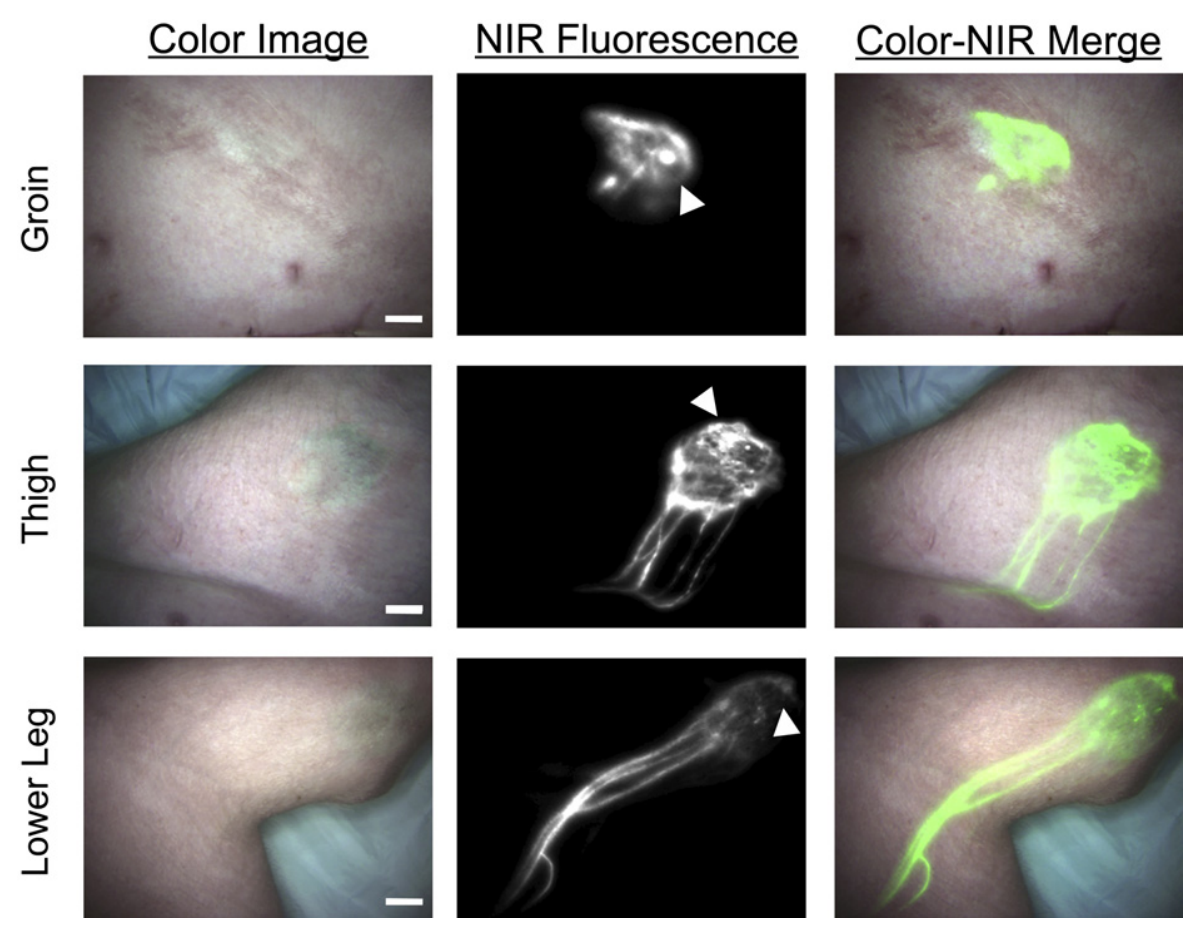

FIGURE E1. Thoracic duct imaging during open surgery in pigs. Lymphatic flow away from the injection site for $71 \mu \mathrm{g} / \mathrm{kg}$ of indocyanine green injected subcutaneously into the groin (top row), thigh (middle row), and lower leg (bottom row). Shown are color video images (left), near-infrared (NIR) fluorescence images (middle), and a pseudocolored (lime green) merge (right). Arrowhead indicates the injection site. Scale bar represents $1 \mathrm{~cm}$. 


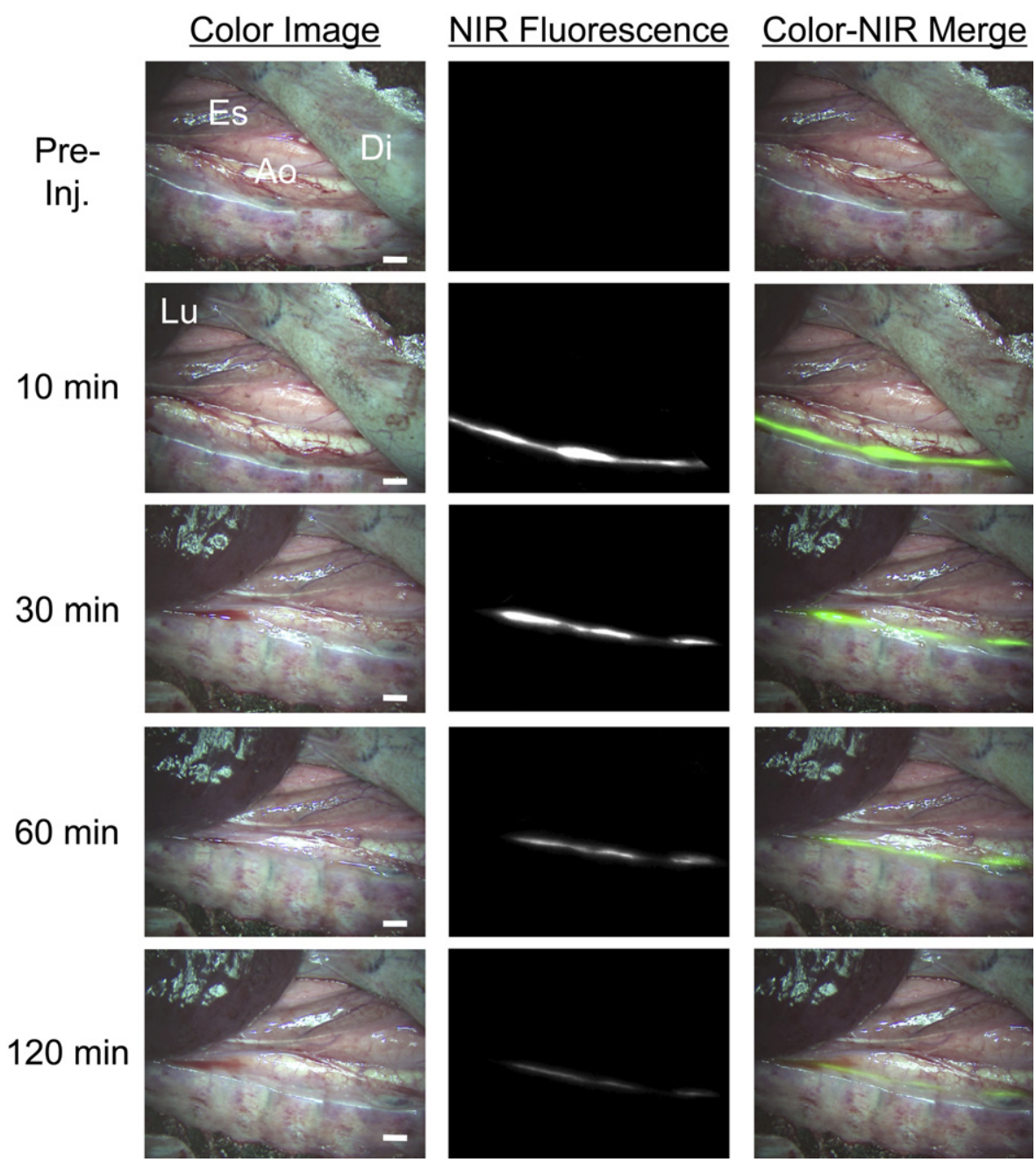

FIGURE E2. Thoracic duct imaging during open surgery in pigs. Visualization of the thoracic duct over time in pigs after subcutaneous injection of $71 \mu \mathrm{g} / \mathrm{kg}$ of indocyanine green into the lower leg. Shown are representative images from before injection (Pre-Inj.) through 120 minutes after injection: Color video (left), near-infrared (NIR) fluorescence (middle), and a pseudocolored (lime green) merge (right). Scale bar represents $1 \mathrm{~cm}$. 